\title{
Changing indications and surgical techniques for keratoplasty during a 16-year period (2003-2018) at a tertiary referral hospital in Japan
}

This article was published in the following Dove Press journal: Clinical Ophthalmology

\section{Tsubasa Nishino \\ Akira Kobayashi \\ Hideaki Yokogawa \\ Natsuko Mori \\ Kazuhisa Sugiyama}

Department of Ophthalmology, Kanazawa University Graduate School of Medical Science, Kanazawa, Japan
Correspondence: Akira Kobayashi Department of Ophthalmology, Kanazawa University Graduate School of Medical Science, I3-I Takara-machi, Kanazawa-shi, Ishikawa-ken, +920-864I, Japan

Tel +8I 762652403

Fax +81 762229660

Email eyekoba@gmail.com
Purpose: To present the changing indications and surgical techniques for keratoplasty during a 16-year period (2003-2018) at a tertiary referral hospital in Japan.

Methods: Consecutive keratoplasty cases at Kanazawa University Hospital from January 2003 to December 2018 were retrospectively reviewed. Keratoplasty procedures included penetrating keratoplasty (PK), deep anterior lamellar keratoplasty (DALK), anterior lamellar keratoplasty (ALK), Descemet's stripping automated endothelial keratoplasty (DSAEK), and Descemet's membrane endothelial keratoplasty (DMEK). Annual numbers and types of keratoplasty as well as underlying diseases for PK and total keratoplasty procedures were recorded, and annual trends were statistically analyzed using Cochran-Armitage test for trend.

Results: A total of 801 keratoplasty procedures (PK, 319 cases; DALK, 57 cases; ALK, 9 cases; DSAEK, 371 cases; and DMEK 45 cases; mean age, $66.9 \pm 16.3$ years) were performed for 595 patients ( 302 males [ 329 eyes, 419 cases], 293 females [ 345 eyes, 382 cases]) during the 16-year period. The proportion of PK procedures decreased significantly in the beginning and showed a slightly increasing trend after a plateau around 2015. DSAEK was increasing after 2006 and reached a plateau around 2012. Among 10 underlying diseases for total keratoplasty, corneal opacity and dermoid were decreasing linearly. Failed PK and failed DSAEK were increasing linearly in the beginning and reached a plateau followed by a decreasing trend. In terms of the underlying disease for PK, bullous keratopathy was decreasing in the beginning and reached a plateau around 2015. A total of 19 PK procedures were performed on cases with recalcitrant bullous kerstopathy (BK) after 2010.

Conclusion: The distribution of keratoplasty procedures and underlying diseases changed significantly over 16 years at a tertiary referral hospital in Japan. PK procedure was significantly decreased and DSAEK procedure was significantly increased. PK for BK decreased significantly; however, PK remains a viable option for other recalcitrant corneal diseases.

Keywords: Descemet's stripping automated endothelial keratoplasty, Descemet's membrane endothelial keratoplasty, penetrating keratoplasty

\section{Introduction}

To date, keratoplasty is the most frequently performed organ transplantation procedure in Japan. ${ }^{1}$ Penetrating keratoplasty (PK) is the original type of keratoplasty procedure, with a history of more than 100 years. In the past decade, the number of lamellar keratoplasty procedures performed has significantly increased compared with PK; lamellar keratoplasty procedures include deep anterior lamellar keratoplasty (DALK), anterior lamellar 
keratoplasty (ALK), and endothelial keratoplasty (EK) such as Descemet's stripping automated endothelial keratoplasty $(\text { DSAEK })^{2-4}$ and Descemet's membrane endothelial keratoplasty (DMEK). ${ }^{5-10}$ Recent statistics have shown that EK procedures are the most commonly performed keratoplasty procedures in the US for the treatment of bullous keratopathy (BK). ${ }^{11}$ In western countries, the majority of underlying diseases for which BK is performed are Fuchs' endothelial dystrophy (FED) ${ }^{9}$ and cataract surgery. ${ }^{11,12}$ In contrast, the results of a national study in Japan conducted between 1999 and 2001 indicated that the underlying diseases for which BK is performed are quite different from those in western countries. ${ }^{13}$ While BK for FED is relatively rare, BK after argon laser iridotomy (ALI) and BK after cataract or glaucoma surgery are commonly performed in Japan. ${ }^{13}$

We previously investigated recent trends in underlying diseases for EK procedures in Japan, ${ }^{14}$ and revealed a significant increase in re-DSAEK and DMEK among all EK procedures. Additionally, among the underlying diseases, the incidence of BK after ALI and FED has decreased and the incidence of BK after trabeculectomy and failed DSAEK has increased in the past decade.

In this study, we further elucidated trends and causal diseases for all keratoplasty procedures, including both PK and other lamellar surgeries, over a much longer period (16 years). It may be beneficial for Asian corneal surgeons or western corneal surgeons treating Japanese and/or Asian patients to understand current trends in keratoplasty procedures at a tertiary referral hospital in Japan.

\section{Methods}

This study was approved by the Medical Ethics Committee of Kanazawa University (approval number, 2645), and followed the tenets of the Declaration of Helsinki. Written informed consent was obtained from all patients.

Charts of patients with corneal diseases surgically treated with keratoplasty by three experienced corneal surgeons (A.K., H.Y., and K.S.) at Kanazawa University Hospital from January 2003 to December 2018 were retrospectively reviewed. Data regarding the annual number and type of keratoplasty procedures and underlying diseases for PK and all keratoplasty procedures during this period were collected. Keratoplasty procedures included PK, DALK, ALK, DSAEK, and DMEK. DSAEK and DMEK were introduced to our hospital in 2006 and 2010, respectively. The following diseases were recorded as underlying diseases for PK: BK, keratoconus, corneal dystrophy, corneal infection, corneal opacity other than dystrophy or ongoing infection or BK, corneal perforation, failed PK, and failed DSAEK. Dermoid and failed DMEK were also recorded as underlying diseases for all keratoplasty procedures. Graft failure after PK/DSAEK was defined as failed PK or failed DSAEK, respectively, independent of BK. If both eyes had undergone keratoplasty, both were enrolled into the study. If a single eye had undergone multiple keratoplasty procedures, each procedure was recorded independently. Trends in keratoplasty procedures and underlying diseases for PK and all keratoplasty procedures were statistically analyzed.

\section{Statistical methods}

To detect monotonic annual trend in causal disease and surgical procedure of corneal endothelial transplantation during study period, Cochran-Armitage test was applied to the 10 diseases (BK, keratoconus, dystrophy, corneal opacity, corneal perforation, corneal infection, failed PK, failed DSAEK, failed DMEK, and dermoid) and the 5 procedures (PK, DALK, ALK, DSAEK, and DMEK). The study period is from 2003 to 2018. However, DSAEK and DMEK were not feasible at 2003, for that reason, baseline years for failed DSAEK and failed DMEK were assumed to be 2006 and 2010, respectively. If $P$-value to the null hypothesis, i.e., no monotonic annual trend exists, was $<0.05$, the disease/procedure was considered to have a significant monotonic increasing or decreasing trend over the years.

Moreover, to fit a smoothed curve for the proportion over years, logistic regression model was applied to the 10 diseases and the 5 procedures. Four types of model were prepared, depending on the highest degree of year term in the explanatory variables, from linear to quartic. For excluding models with significant lack-of-fit to the data, Hosmer-Lemshow test was used. Akaike's information criteria were used to select the best model for each disease/procedure, after excluding models with significant lack-of-fit.

In addition, for evaluation of causal disease in the PK subgroup, similar statistical analyses were performed for eight diseases (BK, keratoconus, dystrophy, corneal opacity, corneal perforation, corneal infection, failed PK, and failed DSAEK).

\section{Results}

A total of 801 keratoplasty procedures (PK, 319 cases; DALK, 57 cases; ALK, 9 cases; DSAEK, 371 cases; and DMEK, 45 cases; mean age, $66.9 \pm 16.3$ years) were performed 
for 595 patients ( 302 males [ 329 eyes, 419 cases], 293 females [345 eyes, 382 cases]) during the 16-year period (Table 1). Total annual keratoplasty numbers gradually increased from 20 cases in 2003 to 69 cases in 2017 and 52 cases in 2018 . Approximately, 40-70 keratoplasty procedures were performed from 2004 to 2018 (Figure 1).

Among the five types of keratoplasty procedures, significant trends were identified for three procedures: PK, DALK and DSAEK. Although the overall number of keratoplasty procedures remained relatively stable from 2004 to 2018, the proportion of PK procedures decreased significantly in the beginning, and showed slightly increasing trend after a plateau around 2015. On the other hand, DALK showed a fluctuating change with two peaks

Table I Total number of keratoplasty procedures from 2003 to 2018

\begin{tabular}{|l|l|l|}
\hline Procedures & No. of cases & Percentage \\
\hline DSAEK & 371 & 46.3 \\
PK & 319 & 39.8 \\
DALK & 57 & 7.1 \\
DMEK & 45 & 5.6 \\
ALK & 9 & 1.1 \\
Total & 801 & \\
\hline
\end{tabular}

Abbreviations: ALK, anterior lamellar keratoplasty; DALK, deep anterior lamellar keratoplasty; DMEK, Descemet's membrane endothelial keratoplasty; DSAEK, Descemet's stripping automated endothelial keratoplasty; PK, penetrating keratoplasty. around 2005 and 2014. DSAEK was increasing after 2006 and reached a plateau around 2012. (Figure 2, Table S1).

A total of 319 PK procedures were performed for patients with eight types of corneal disease during the 16-year period (Table 2). In terms of the underlying disease for PK, significant monotonic trends over years were observed in 5 diseases, i.e., bullous keratoplasty, keratoconus, perforation, failed PK, and failed DSAEK (Figure 3, Table S2). BK was decreasing in the beginning, and showed increasing trend after a plateau around 2015. Keratoconus was increasing linearly. Perforation was increasing in the beginning, reached a plateau around 2009, and then showed an increasing trend in the last part of study period. Failed PK was increasing in the beginning, and showed a decreasing trend after a peak around 2013. Failed DSAEK was observed in 2016 for the first time.

Ten underlying diseases for keratoplasty, BK, keratoconus, dystrophy, corneal opacity, corneal perforation, corneal infection, failed PK, failed DSAEK, failed DMEK, and dermoid, were evaluated in this study. BK was the most common cause of keratoplasty throughout the 16-year period (440 cases, 54.9\%; Table 3). Significant monotonic trends over the years were observed in 5 diseases, i.e., dystrophy, corneal opacity, failed PK, failed DSAEK, and, dermoid (Figure 4, Table S3). Corneal opacity and dermoid were decreasing linearly. Failed PK and failed DSAEK were

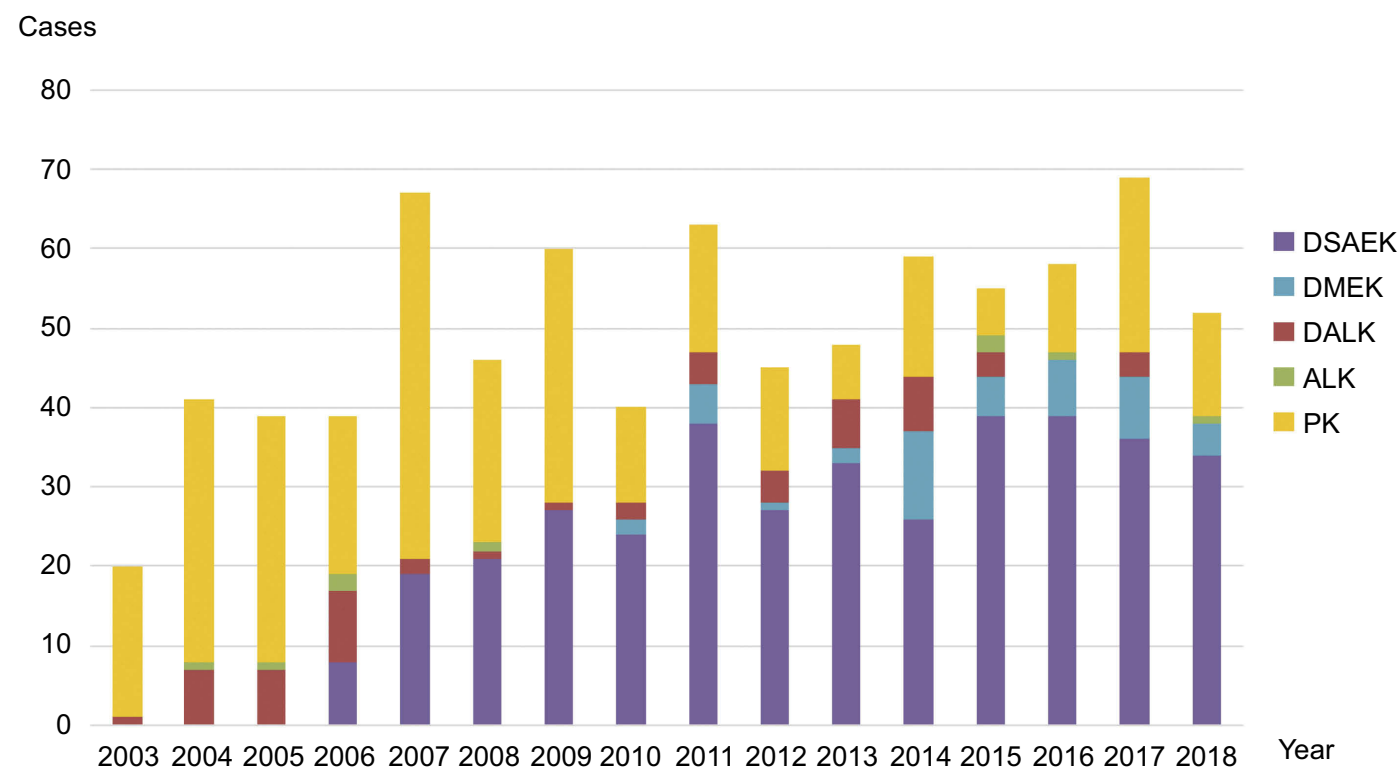

Figure I Number of keratoplasty procedures by year. The total annual number of keratoplasty procedures increased from 20 in 2003 to 52 in 2018 . PK and DSAEK have consistently remained as major keratoplasty types.

Abbreviations: DSAEK, Descemet's stripping automated endothelial keratoplasty; DMEK, Descemet's membrane endothelial keratoplasty; DALK, deep anterior lamellar keratoplasty; ALK, anterior lamellar keratoplasty; PK, penetrating keratoplasty. 


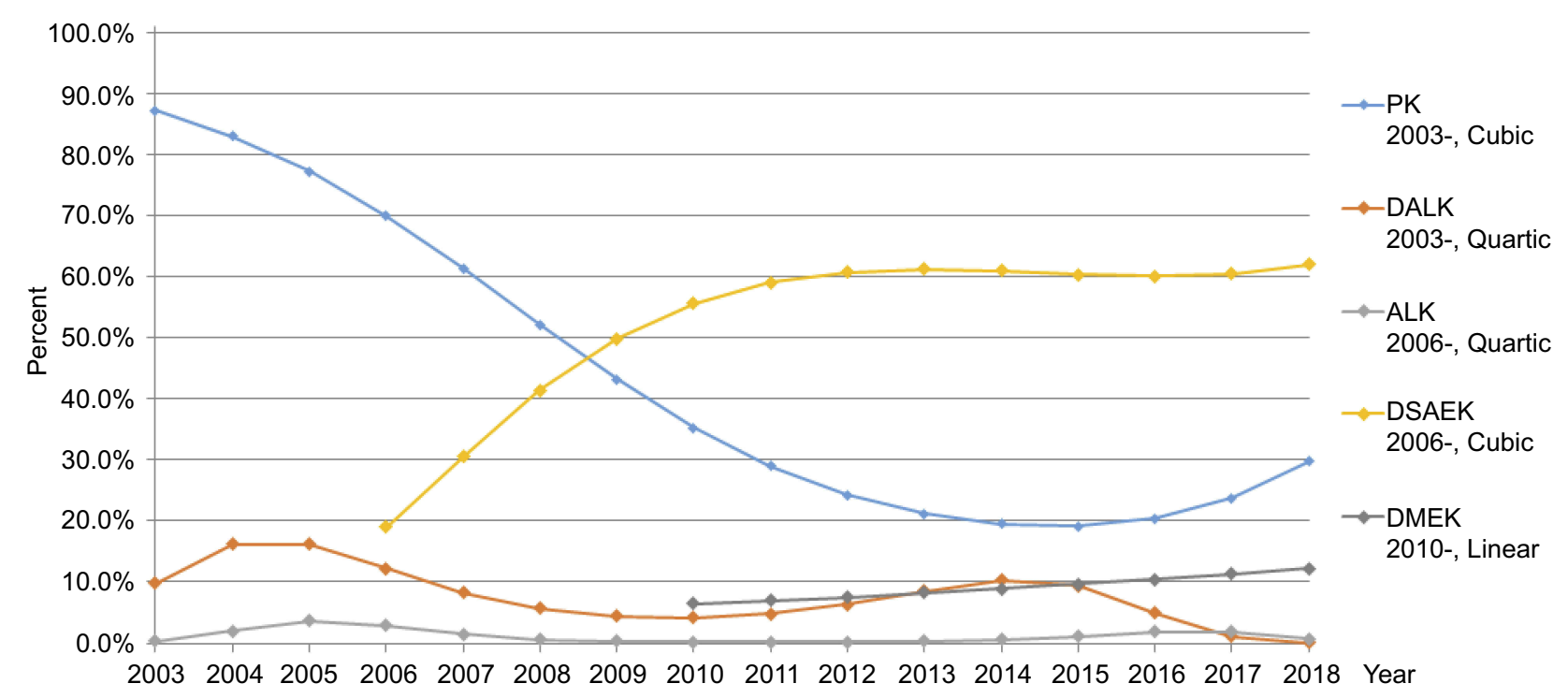

Figure 2 Trends in keratoplasty procedures from 2003 to 2018. The incidence of PK procedures decreased, while the incidence of DSAEK significantly increased. Abbreviations: DSAEK, Descemet's stripping automated endothelial keratoplasty; DMEK, Descemet's membrane endothelial keratoplasty; DALK, deep anterior lamellar keratoplasty; ALK, anterior lamellar keratoplasty; PK, penetrating keratoplasty.

Table 2 Underlying disease indications for PK from 2003 to 2018

\begin{tabular}{|l|l|l|}
\hline Underlying disease & No. of cases & Percentage \\
\hline Bullous keratopathy & 120 & 37.6 \\
Corneal opacity & 80 & 25.1 \\
Keratoconus & 43 & 13.5 \\
Perforation & 28 & 8.7 \\
Infection & 20 & 6.3 \\
Failed PK & 19 & 6.0 \\
Dystrophy & 7 & 2.1 \\
Failed DSAEK & 2 & 0.6 \\
Total & 319 & \\
\hline
\end{tabular}

Abbreviations: DSAEK, Descemet's stripping automated endothelial keratoplasty; PK, penetrating keratoplasty.

increasing linearly in the beginning of the study period, but in the latter half, reached a plateau. Dystrophy showed a fluctuating change with a peak in 2006 and was increasing in the last part of the study period.

\section{Discussion}

In this study, we retrospectively reviewed total keratoplasty procedures (PK, DALK, ALK, DSAEK, and DMEK) performed over a 16-year period (2003-2018) at a tertiary referral hospital in Japan to observe trends in surgical procedures and causal diseases. During this period, 801 keratoplasties were performed: PK, 309 cases (38.6\%); DALK, 57 cases (7.1\%); ALK, 9 cases (1.1\%); DSAEK, 371 cases (46.3\%); and DMEK, 45 cases $(5.6 \%)$.
The analysis revealed a significant increase in DSAEK procedures and a significant decrease in PK procedures. DALK showed a fluctuating change with two peaks around 2005 and 2014. Although DMEK did not show increase with statistical significance, it tends to increase after the introduction in 2010. The increased popularity of DSEAK in Japan (as well as in other countries) may be due to its many advantages, including superior visual acuity with less astigmatism, rapid visual rehabilitation, resistance to trauma, and the ability to make small corneal incisions with no stroma incisions/sutures. This trend is consistent with that observed in the US ${ }^{11}$ It should also be noted that the development of precut DSAEK tissue ${ }^{15}$ or prestripped DMEK tissue ${ }^{16}$ during this period has greatly shortened operation time, eliminating the need for a large capital investment in an expensive microkeratome machine for DSAEK, and preventing tissue loss due to unsuccessful Descemet's membrane stripping/harvesting during DMEK.

The causal diseases for keratoplasty in Japan have been previously reported to be completely different from those in western countries. ${ }^{12-14}$ In a national survey of BK procedures in Japan from 1999 to 2001 conducted by Shimazaki et al, BK accounted for $24.2 \%$ (963 eyes) of total keratoplasty procedures. Cataract surgery was the most common cause of BK ( $n=428,44.4 \%)$, followed by BK secondary to laser iridotomy (LI) $(n=225,23.4 \%)$. In contrast to western countries, in which FED comprises the 


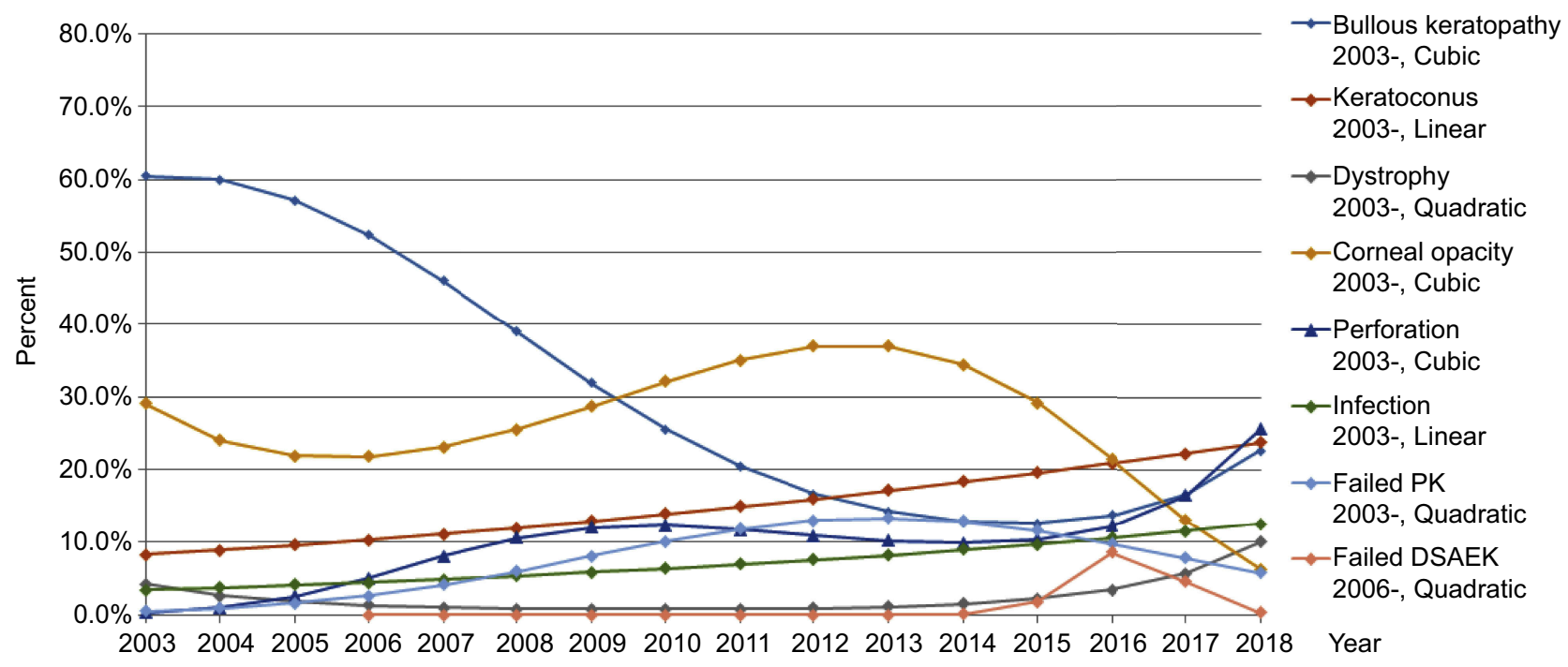

Figure 3 Trends in underlying diseases for PK from 2003 to 2018 . The incidence of BK significantly decreased.

Abbreviations: BK, bullous kerstopathy; DSAEK, Descemet's stripping automated endothelial keratoplasty; PK, penetrating keratoplasty.

Table 3 Underlying disease indications for all keratoplasty procedures from 2003 to 2018

\begin{tabular}{|l|l|l|}
\hline Underlying disease & No. of cases & Percentage \\
\hline Bullous keratopathy & 440 & 54.9 \\
Corneal opacity & 110 & 13.7 \\
Keratoconus & 56 & 7.0 \\
Failed DSAEK & 55 & 6.9 \\
Failed PK & 53 & 6.6 \\
Perforation & 37 & 4.6 \\
Infection & 20 & 2.5 \\
Dystrophy & 14 & 1.7 \\
Failed DMEK & 9 & 1.1 \\
Dermoid & 7 & 0.9 \\
Total & 801 & \\
\hline
\end{tabular}

Abbreviations: DMEK, Descemet's membrane endothelial keratoplasty; DSAEK, Descemet's stripping automated endothelial keratoplasty; PK, penetrating keratoplasty.

majority of BK cases, FED was the cause of BK in only 18 eyes (1.9\%) in Japan. Recently, we reported the changing indications and procedures for endothelial keratoplasty (DSAEK and DMEK) over a 10-year period (2007 to 2016), including a significant increase in the incidence of these procedures. ${ }^{14}$ With respect to causal diseases for BK, BK secondary to LI was the leading cause in 2007, followed by FED and failed PK. In 2016, BK after trabeculectomy was most prevalent, followed by failed DSAEK, failed PK, and pseudophakic BK. The decreased incidence of ALI and FED and increased incidence of BK after trabeculectomy and failed DSAEK were statistically significant. ${ }^{14}$ These results confirmed the differences in causal diseases for BK in Japan compared with those of western countries.

In this study, we analyzed current trends in PK procedures. Although the incidence of PK has decreased, it remains as an important procedure for some patients. As causal diseases for PK during the 16-year period, the incidence of BK was decreasing in the beginning and reached a plateau around 2015. A total of 19 PK procedures were performed on cases with BK after 2010 in our hospital: 8 cases with BK after globe rupture, 3 cases with BK due to microcornea, and others with BK after multiple surgeries or with proliferative vitreoretinopathy. All of these complicated BK cases had an excellent postoperative course with no endothelial decompensation for 3-year follow up, indicating that PK remains a viable and important option for other recalcitrant corneal diseases.

A limitation of the current analysis is that it is a singlecenter study, so the results obtained herein may not necessarily reflect the overall trends in Japan. Nonetheless, this study has successively elucidated recent trends in keratoplasty procedures during a 16 -year period in a tertiary referral hospital in Japan.

In conclusion, the distribution of keratoplasty procedures (PK/DALK/ALK/DSAEK/DMEK) and underlying diseases changed significantly over a 16-year period (2003 to 2008) at a tertiary referral hospital in Japan. A significant decrease in PK procedures and a significant increase in DSAEK procedures were observed. The use of PK for BK decreased significantly; however, PK remains a viable option for the 


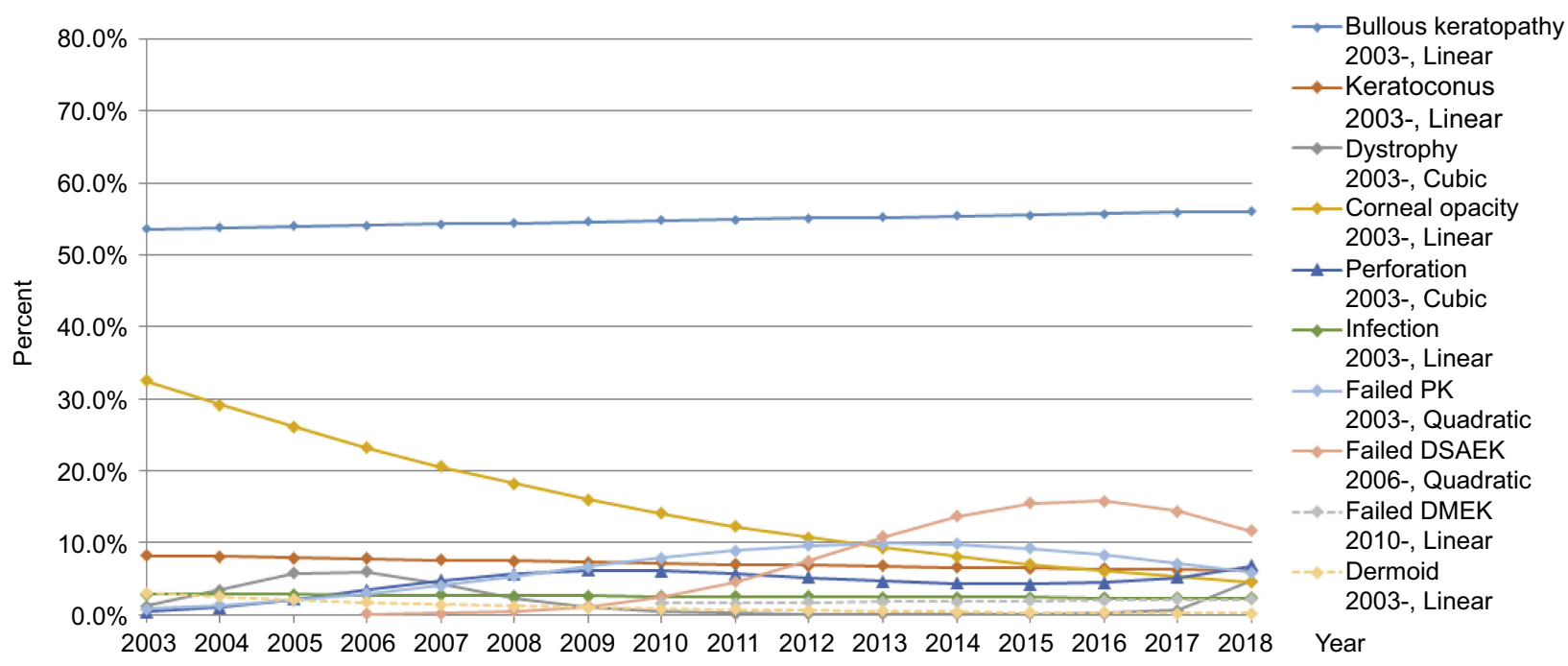

Figure 4 Trends in underlying diseases for all keratoplasty procedures from 2003 to 2018 . Corneal opacity and dermoid were decreasing linearly. Failed PK and failed DSAEK were increasing linearly in the beginning of the study period, but in the latter half, reached a plateau.

Abbreviations: DSAEK, Descemet's stripping automated endothelial keratoplasty; DMEK, Descemet's membrane endothelial keratoplasty; PK, penetrating keratoplasty.

treatment of other recalcitrant corneal diseases. The results obtained herein may be beneficial for Asian corneal surgeons or western corneal surgeons treating Japanese and/or Asian patients, as understanding very recent trends in keratoplasty procedures may better inform the selection of surgical procedure. Further, multicenter studies are required to fully understand the trends in keratoplasty procedures throughout Japan.

\section{Acknowledgment}

This study was supported by Grant-in-Aid for Scientific Research (C) KAKENHI, Japan (No. 19K09947).

\section{Disclosure}

The authors report no conflicts of interest in this work.

\section{References}

1. Report on the status of organ transplantation. Available from: https:// www.mhlw.go.jp/file/04-Houdouhappyou-10905500-KenkoukyokuZoukiishokushitsu/0000165996.pdf. Accessed July 22, 2019 (in Japanese).

2. Melles GR, Eggink FA, Lander F, et al. A surgical technique for posterior lamellar keratoplasty. Cornea. 1998;17:618-626.

3. Terry MA, Ousley PJ. Small-incision deep lamellar endothelial keratoplasty (DLEK): six-month results in the first prospective clinical study. Cornea. 2005;24:59-65.

4. Gorovoy MS. Descemet-stripping automated endothelial keratoplasty. Cornea. 2006;25:886-889. doi:10.1097/01.ico.0000214224.90743.01

5. Melles GR, Lander F, Rietveld FJ. Transplantation of Descemet's membrane carrying viable endothelium through a small scleral incision. Cornea. 2002;21:415-418. doi:10.1097/00003226-200205000-00016
6. Melles GR, Ong TS, Ververs B, van der Wees J. Descemet's membrane endothelial keratoplasty (DMEK). Cornea. 2006;25:987-990. doi:10.1097/01.ico.0000248385.16896.34

7. Melles GR. Posterior lamellar keratoplasty: DLEK to DSEK to DMEK. Cornea. 2006;25:879-881. doi:10.1097/01.ico.0000243962.60392.4f

8. Ham L, Balachandran C, Verschoor CA, et al. Visual rehabilitation rate after isolated Descemet membrane transplantation: Descemet membrane endothelial keratoplasty. Arch Ophthalmol. 2009;127:252-255. doi:10.1001/archophthalmol.2008.619

9. Price MO, Giebel AW, Fairchild KM, et al. Descemet's membrane endothelial keratoplasty; prospective multi-center study of visual and refractive outcomes and endothelial survival. Ophthalmology. 2009;116:2361-2368. doi:10.1016/j.ophtha.2009.07.010

10. Anshu A, Price MO, Price FW Jr. Risk of corneal transplant rejection significantly reduced with Descemet's membrane endothelial keratoplasty. Ophthalmology. 2012;119:536-540. doi:10.1016/j.ophtha.2011.09.019

11. Eye Bank Association of America. 2017. Eye banking statistical report. Available from: www.restoresight.org. Accessed July 22, 2019.

12. Dobbins KR, Price FW Jr, Whitson WE. Trends in the indications for penetrating keratoplasty in the midwestern United States. Cornea. 2000;19:813-816.

13. Shimazaki J, Amano S, Uno T, Maeda N; The Japan Bullous Keratopathy Study Group. National survey on bullous keratopathy in Japan. Cornea. 2007;26:274-278. doi:10.1097/ICO.0b013e31802c9e19

14. Nishino T, Kobayashi A, Yokogawa H, et al. A 10-year review of underlying diseases for endothelial keratoplasty (DSAEK/DMEK) in a tertiary referral hospital in Japan. Clin Ophthalmol. 2018;12:13591365. doi: $10.2147 /$ OPTH.S170263

15. Terry MA, Shamie N, Chen ES, et al. Precut tissue for Descemet's stripping automated endothelial keratoplasty: vision, astigmatism, and endothelial survival. Ophthalmology. 2009;116:248-256. doi:10.1016/ j.ophtha.2008.09.017

16. Kobayashi A, Murata N, Yokogawa H, Yamazaki N, Masaki T, Sugiyama K. Evaluation of internationally shipped prestripped donor tissue for descemet membrane endothelial keratoplasty by vital dye staining. Cornea. 2015;34:225-227. doi:10.1097/ICO.000000000000 0330 


\section{Supplementary materials}

Table SI Trends in total keratoplasty procedures from 2003 to 2018 using logistic regression model analysis and Cochran-Armitage test

\begin{tabular}{|c|c|c|c|c|c|c|c|}
\hline \multirow{2}{*}{$\begin{array}{l}\text { Keratoplasty } \\
\text { procedures }\end{array}$} & \multirow{2}{*}{$\begin{array}{l}\text { Baseline } \\
\text { Year } \\
\text { Model }\end{array}$} & \multirow[t]{2}{*}{ Parameter } & \multicolumn{3}{|c|}{ Logisitic model parameter estimate } & \multirow{2}{*}{$\begin{array}{l}\text { Hosmer- } \\
\text { Lemeshow } \\
\text { goodness-of-Fit } \\
\text { test }\end{array}$} & \multirow{2}{*}{$\begin{array}{l}\text { Cochran-Armitage } \\
\text { test for } \\
\text { Monotonicity }\end{array}$} \\
\hline & & & Estimate & 95\% C.I. & $P$-value & & \\
\hline PK & $\begin{array}{l}2003 \\
\text { Cubic }\end{array}$ & $\begin{array}{l}\text { Intercept at BL } \\
\text { Linear trend } \\
\text { Quadratic trend } \\
\text { Cubic trend }\end{array}$ & $\begin{array}{l}1.917 \\
-0.314 \\
-0.020 \\
0.002\end{array}$ & $\begin{array}{l}(1.117,2.717) \\
(-0.710,0.082) \\
(-0.077,0.037) \\
(0.000,0.004)\end{array}$ & $\begin{array}{l}- \\
0.121 \\
0.490 \\
0.116\end{array}$ & $P=0.105$ & $P<0.00 I^{* *}$ \\
\hline DALK & $\begin{array}{l}2003 \\
\text { Quartic }\end{array}$ & $\begin{array}{l}\text { Intercept at BL } \\
\text { Linear trend } \\
\text { Quadratic trend } \\
\text { Cubic trend } \\
\text { Quartic trend }\end{array}$ & $\begin{array}{l}-2.218 \\
0.967 \\
-0.444 \\
0.056 \\
-0.002\end{array}$ & $\begin{array}{l}(-3.403,-1.034) \\
(-0.230,2.164) \\
(-0.804,-0.083) \\
(0.017,0.096) \\
(-0.004,-0.001)\end{array}$ & $\begin{array}{l}- \\
0.113 \\
0.016 * * \\
0.005 * * \\
0.003 * *\end{array}$ & $P=0.068$ & $P=0.00 I^{* *}$ \\
\hline ALK & $\begin{array}{l}2003 \\
\text { Quartic }\end{array}$ & $\begin{array}{l}\text { Intercept at BL } \\
\text { Linear trend } \\
\text { Quadratic trend } \\
\text { Cubic trend } \\
\text { Quartic trend }\end{array}$ & $\begin{array}{l}-5.746 \\
2.670 \\
-0.887 \\
0.091 \\
-0.003\end{array}$ & $\begin{array}{l}(-9.892,-1.601) \\
(-1.037,6.378) \\
(-1.906,0.132) \\
(-0.010,0.191) \\
(-0.006,0.000)\end{array}$ & $\begin{array}{l}- \\
0.158 \\
0.088 \\
0.077 \\
0.080\end{array}$ & $P=0.345$ & $P=0.550$ \\
\hline DSAEK & $\begin{array}{l}2006 \\
\text { Cubic }\end{array}$ & $\begin{array}{l}\text { Intercept at BL } \\
\text { Linear trend } \\
\text { Quadratic trend } \\
\text { Cubic trend }\end{array}$ & $\begin{array}{l}-1.450 \\
0.709 \\
-0.086 \\
0.003\end{array}$ & $\begin{array}{l}(-2.038,-0.86 \mathrm{I}) \\
(0.297,1.12 \mathrm{I}) \\
(-0.164,-0.008) \\
(-0.001,0.008)\end{array}$ & $\begin{array}{l}- \\
<0.001 * * \\
0.030 * * \\
0.112\end{array}$ & $P=0.153$ & $P<0.00 I^{* *}$ \\
\hline DMEK & $\begin{array}{l}2010 \\
\text { Linear }\end{array}$ & $\begin{array}{l}\text { Intercept at BL } \\
\text { Linear trend }\end{array}$ & $\begin{array}{l}-2.681 \\
0.088\end{array}$ & $\begin{array}{l}(-3.334,-2.028) \\
(-0.035,0.212)\end{array}$ & $\begin{array}{l}- \\
0.161\end{array}$ & $P=0.130$ & $P=0.159$ \\
\hline
\end{tabular}

\footnotetext{
Note: **Statistically significant.
}

Abbreviations: ALK, anterior lamellar keratoplasty; DALK, deep anterior lamellar keratoplasty; DMEK, Descemet's membrane endothelial keratoplasty; DSAEK, Descemet's stripping automated endothelial keratoplasty; PK, penetrating keratoplasty. 


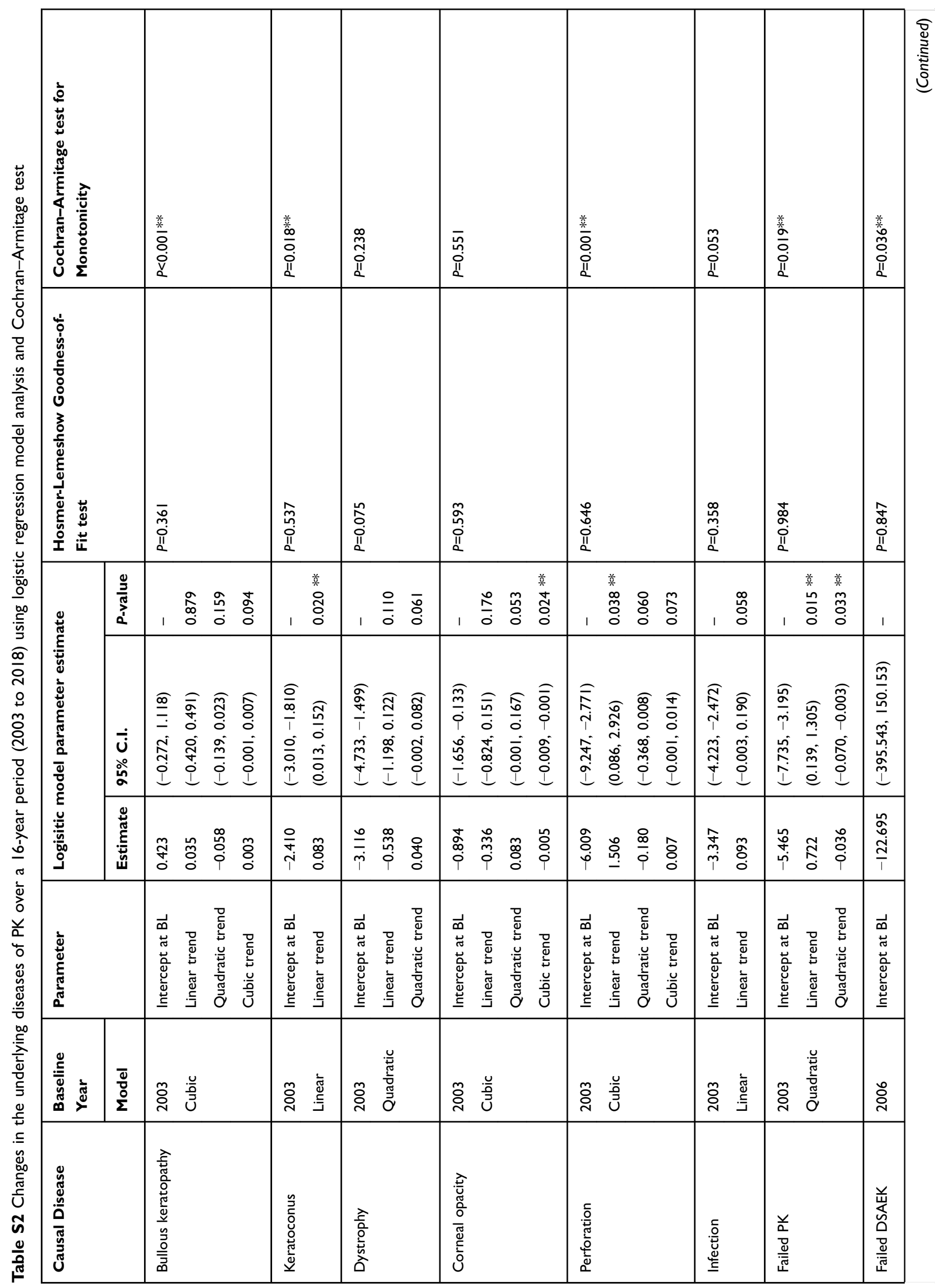




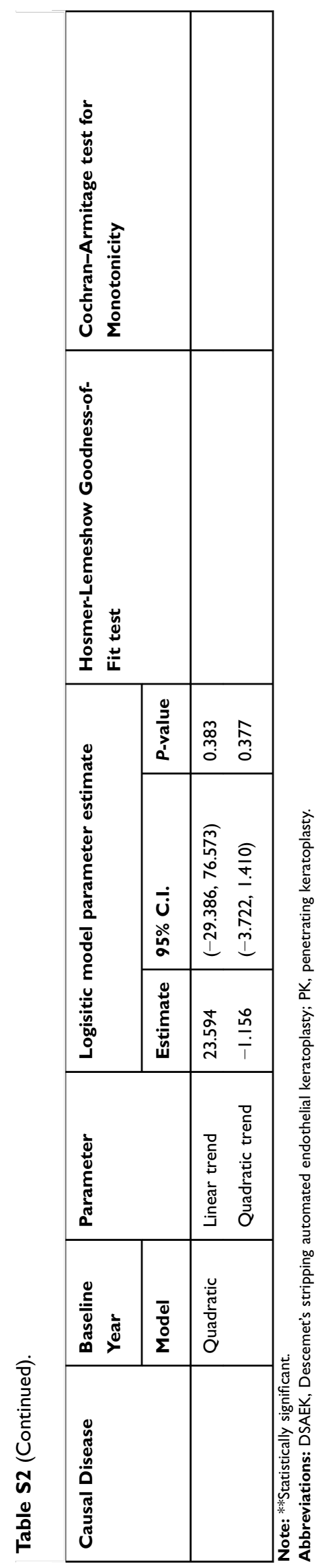




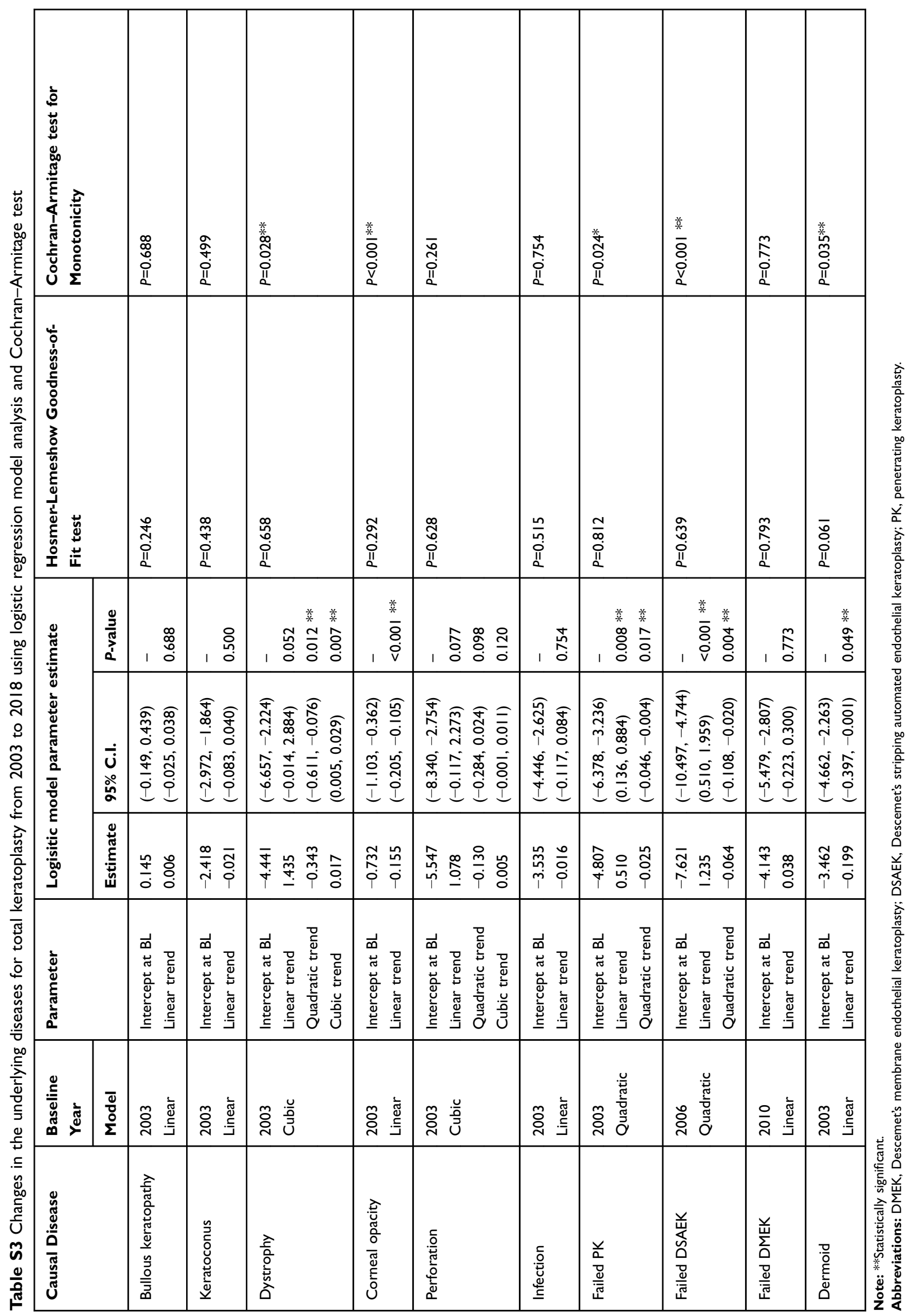




\section{Publish your work in this journal}

Clinical Ophthalmology is an international, peer-reviewed journal covering all subspecialties within ophthalmology. Key topics include: Optometry; Visual science; Pharmacology and drug therapy in eye diseases; Basic Sciences; Primary and Secondary eye care; Patient Safety and Quality of Care Improvements. This journal is indexed on PubMed

Central and CAS, and is the official journal of The Society of Clinical Ophthalmology (SCO). The manuscript management system is completely online and includes a very quick and fair peer-review system, which is all easy to use. Visit http://www.dovepress.com/ testimonials.php to read real quotes from published authors. 